\title{
Cosmological dynamics of $f(R)$ models in dynamical system analysis
}

\author{
Parth Shah ${ }^{1}$ and Gauranga C. Samanta 2 :*, \\ ${ }^{1}$ Department of Mathematics, BITS Pilani K K Birla Goa Campus, Goa, India \\ ${ }^{2}$ P. G. Department of Mathematics, Fakir Mohan University, Odisha, India \\ parthshah2908@gmail.com \\ gauranga81@gmail.com
}

\begin{abstract}
In this work we try to understand the late time acceleration of the universe by assuming some modification in the geometry of the space and using dynamical system analysis. This technique allows to understand the behavior of the universe without analytically solving the field equations. We study the acceleration phase of the universe and stability properties of the critical points which could be compared with observational results. We consider an asymptotic behavior of two particular models $f(R)=R-\mu R_{c} \frac{\left(R / R_{c}\right)^{2 n}}{\left(R / R_{c}\right)^{2 n}+1}$ and $f(R)=R-\mu R_{c}\left[1-\left(1+R^{2} / R_{c}^{2}\right)^{-n}\right]$ with $n, \mu, R_{c}>0$ for the study. As a first case we fix the value of $\mu$ and analyzed for all $n$. Later as second case, we fix the value of $n$ and calculation are done for all $\mu$. At the end all the calculations for the generalized case have been shown and results have been discussed in detail.
\end{abstract}

Keywords: Dark energy, Modified gravity theory, Dynamical system analysis

\section{Introduction}

More than a century ago, Einstein proposed his theory of general relativity (GR) which revolutionized the idea of gravity. It is a theory that acted as a powerful tool in our pursuit to understand the universe. With the passage of time and with extensive research, snags in the theory started showing up. The major blow came towards the end of the last century when the accelerated expansion of the universe [1,2] was discovered which left GR inconsistent at the cosmological distances. Since then researchers have started looking for the alternative techniques such as modified gravity and dark energy to incorporate this accelerated expansion in the theory of gravity. While the former deals with the geometry of spacetime, the latter is concerned with the matter content of the universe. These are two different approaches to understand the late time acceleration of the universe. Extensive reviews in modified gravity can be found in the Refs. [3 5]. Many of such theories aim at modifying the linear function of scalar curvature $R$ from its special form in GR to a more generic form. $f(R)$ gravity is one such attempt where the gravitational lagrangian of GR, $\mathcal{L}_{G R}=R$ is replaced by an analytic function of $R$ i.e. $\mathcal{L}_{f(R)}=f(R)$. Choosing a suitable function for $f(R)$, one can explore the non-linear effects of the scalar curvature on the evolution of the universe. Extensive reviews in $f(R)$ gravity can be found in the refs [6, 7]. Viability of $f(R)$ dark energy models have been studied in ref. [8], where the $f(R)$ models with a power law of $R$ has been ruled out. Author of ref. 9] studied the interplay between $f(R)$ theories and scalar-tensor theories via the Palatini formalism. Formation of large scale structure in $f(R)$ gravity was studied in ref. [10]. A reconstruction scheme for $f(R)$ theories was explored in ref. [11]. Various other studies related to $f(R)$ gravity can be found in [12 15]. There are many ways other ways to modify the general theory of relativity. Some of the other alternatives are Scalar Tensor Theory [16- 23], Brans Dicke theory [24, 25], Gauss Bonnet theory [26], $f(T)$ gravity [27 32] $f(R, T)$ gravity [33, 34], $f(R, G)$ gravity [35], Lovelock gravity [36, 37]. $f(R)$ gravity has been described in detail in [38 50. Subsequently many authors [51 58] studied cosmological models from various aspects in modified gravity.

${ }^{*}$ Corresponding author. 
It is very difficult to find out the analytic as well as numerical solution in general as well as modified theory of gravity. This is due to the fact that field equations contains nonlinear terms and that leads to difficulty in comparison with observations. One such method to avoid this problem and to study the dynamical behavior of these equations is the dynamical system analysis. Many authors have used this approach in cosmology [59 67]. This methods aims at finding the numerical solution of the system which helps to understand the qualitative behavior of the system. First we obtain the critical points of the given set of first order differential equations. Then we apply the linear stability theory which is to linearise the system near the critical point in order to understand the dynamics of the whole system. In this theory only the first partial derivates is considered which corresponds to the Jacobian matrix in vector calculus and is also referred as stability matrix. The eigenvalues of the Jacobian matrix contain the information about the stability of particular critical point. Although this theory fails for non-hyperbolic points for which Lyapunov's method and central manifold theory are used often. Application of dynamical systems analysis to cosmology has been discussed in these books [68] and 69].

In the present work we analyze the stability and acceleration phase of the cosmological model of the universe which is assumed to have modification in its geometry part, i.e. $f(R)$ gravity. We begin with understanding the Metric formalism and Palatini formalism of $f(R)$ gravity. This work has been carried out in Metric formalism. In this work, we consider the universe to be composed of matter and radiation with no interaction between them. We begin by considering two different $f(R)$ models and show that their asymptotic behavior is same. We study the existence of stability phase and acceleration era for various cases by calculations and plots. We conducted this study due to the unique property of two different $f(R)$ models to be an asymptote to a same model. Also we have studied the stability and acceleration based on the all possible values of parameters $\mu$ and $n$ for these models. In section 2 brief review of metric formalism of $f(R)$ theory is discussed, stability analysis and acceleration phase analysis of the model is done is section 3. Section 4 contains results, conclusions and future possible work.

\section{Metric $f(R)$ gravity}

As it is known that this theory comes as a straightforward generalization of the Lagrangian with matter part in the Einstein-Hilbert action,

$$
S_{E H}=\frac{1}{2 \kappa} \int d^{4} x \sqrt{-g} R+S^{(m)}
$$

where $\kappa=8 \pi G, R$ is Ricci Scalar, $g$ is determinant of metric, $g_{\mu \nu}=\operatorname{diag}\left(-1, a^{2}(t), a^{2}(t), a^{2}(t)\right)$ and $a(t)$ is scale factor to become a general function of $R$, i.e.

$$
S=\frac{1}{2 \kappa} \int d^{4} x \sqrt{-g} f(R)+S^{(m)}
$$

where $f(R)$ is a non-linear function of its argument and $S^{(m)}$ is the matter part of the action. Variation of this action in standard metric formalism with respect to the metric $g^{\mu \nu}$ gives

$$
F(R) R_{\mu \nu}-\frac{1}{2} f(R) g_{\mu \nu}+\left[\square g_{\mu \nu}-\nabla_{\mu} \nabla_{\nu}\right] F(R)=\kappa T_{\mu \nu}
$$

where, $F(R)$ (also denoted $\left.f_{, R}\right)$ is $\frac{\partial f}{\partial R}$ and as usual,

$$
T_{\mu \nu}=\frac{-2}{\sqrt{-g}} \frac{\delta S_{m}}{\delta g^{\mu \nu}}
$$

where, $\nabla_{\mu}$ is the covariant derivative associated with the metric and $\square \equiv \nabla^{\mu} \nabla_{\mu}$.

It can be noted from (3) that these are fourth order differential equations in the metric, since $R$ contains 
second order partial derivatives. Theory is reduced to GR when the action contains only $R$ since the last two terms of the left hand side vanishes. The trace of equation (3) is given by

$$
3 \square F(R)+F(R) R-2 f(R)=\kappa^{2} T
$$

We also note that field equations could be written in the form of Einstein equations by moving the effective stress-energy tensor to the right hand side. Specifically, (3) can be re-written as

$$
\begin{aligned}
G_{\mu \nu} & \equiv R_{\mu \nu}-\frac{1}{2} g_{\mu \nu} R \\
& =\frac{\kappa^{2} T_{\mu \nu}}{F(R)}+g_{\mu \nu}\left[\frac{f(R)-R F(R)}{2 F(R)}\right]+\left[\frac{\nabla_{\mu} \nabla_{\nu}-g_{\mu \nu} \square F(R)}{F(R)}\right]
\end{aligned}
$$

or

$$
G_{\mu \nu}=\frac{k}{F(R)}\left(T_{\mu \nu}+T_{\mu \nu}^{(e f f)}\right)
$$

This gives an effective stress-energy tensor which does not have the canonical form quadratic in the first derivatives of the field $f(R)$, but contains terms linear in the second derivatives. Also it was very essential to form set of conditions which are viable for $f(R)$ models in metric formalism. These conditions have been stated and discussed in [12, 38, 39].

\section{Stability Analysis}

In this paper, the following viable $f(R)$ models have been considered to explain cosmic acceleration using dynamical system techniques.

$$
\begin{aligned}
& f(R)=R-\mu R_{c} \frac{\left(R / R_{c}\right)^{2 n}}{\left(R / R_{c}\right)^{2 n}+1} \\
& f(R)=R-\mu R_{c}\left[1-\left(1+R^{2} / R_{c}^{2}\right)^{-n}\right]
\end{aligned}
$$

with $n, \mu, R_{c}>0$ [38, 40, 41]. This model satisfies all the local gravity conditions and are considered to be a viable model to study the stability analysis of the universe. It is noted here in both these models the function $f(R)$ asymptotically behaves as $f(R) \rightarrow R-\mu R_{c}\left[1-\left(R^{2} / R_{c}^{2}\right)^{-n}\right]$ for $R \gg R_{c}$. These models also satisfy $f(R=0)=0$, so the cosmological constant vanishes in the flat space time. These models are chosen as the property that two $f(R)$ models are asymptote to a single $f(R)$ model is very difficult to find. For the flat FLRW space-time the Ricci scalar is given by:

$$
R=6\left(2 H^{2}+\dot{H}\right)
$$

where $H$ is the Hubble parameter. We now construct a model of the universe filled with only matter and radiation and we assume no interaction between them i. e. the usual conservation equations $\rho_{m}+3 H \rho_{m}=$ 0 and $\dot{\rho}_{r}+4 H \rho_{r}=0$. We also assume that matter is pressure-less i.e. $p_{m}=0$. For this, the explicit form of field equations from equation (4) are

$$
\begin{array}{r}
3 F H^{2}=\kappa^{2}\left(\rho_{m}+\rho_{r}\right)+\frac{F R-f}{2}-3 H \dot{F} \\
-2 F \dot{H}=\kappa^{2}\left(\rho_{m}+\frac{4}{3} \rho_{r}\right)+\ddot{F}-H \dot{F}
\end{array}
$$

Now, we would like to convert the above non-autonomous field equations to an autonomous systems by introducing the following dimensionless variables, 


$$
x=-\frac{\dot{F}}{H F}, y=-\frac{f}{6 F H^{2}}, z=\frac{R}{6 H^{2}}, w=\frac{\kappa^{2} \rho_{r}}{3 F H^{2}}
$$

Without loss of generality, let $\kappa^{2}=\frac{8 \pi G}{c^{4}}=1$. Then, the various density parameters would be,

$$
\Omega_{r}=\frac{\rho_{r}}{3 F H^{2}}=w, \Omega_{m}=\frac{\rho_{m}}{3 F H^{2}}=1-x-y-z-w, \Omega_{G C}=x+y+z
$$

where, $\Omega_{G C}$ represents density parameter due to geometric curvature. From equation (10), it is straightforward to derive following set of autonomous differential equations

$$
\begin{aligned}
x^{\prime} & =-1-z-3 y+x^{2}-x z+w \\
y^{\prime} & =\frac{x z}{m}-y(2 z-4-x) \\
z^{\prime} & =-\frac{x z}{m}-2 z(z-2) \\
w^{\prime} & =-2 z w+x w
\end{aligned}
$$

where, prime denotes derivative with respect to $\eta=\ln a$ and

$$
\begin{aligned}
m & \equiv \frac{d \ln F}{d \ln R}=\frac{R f_{, R R}}{f_{, R}} \\
r & \equiv-\frac{d \ln f}{d \ln R}=-\frac{R f_{, R}}{f}=\frac{z}{y}
\end{aligned}
$$

From this, $R$ could be written as a function of $\frac{z}{y}$. We here note that $m$ is a function of $R$, so it follows that $m$ is a function of $r$, i.e. $m=m(r)$. From the calculations for the model, $f(R)=R-\mu R_{c}\left[1-\left(R^{2} / R_{c}^{2}\right)^{-n}\right]$ we deduce

$$
m=\frac{2 n(2 n+1)}{\mu^{2 n}}(-r-1)^{2 n+1}
$$

By linear approximation and substituting value of $r, m$ could be rewritten as:

$$
m=-\frac{2 n(2 n+1)}{\mu^{2 n}}\left(1+(2 n+1) \frac{z}{y}\right)
$$

We analyze these equations in two different cases.

Case A: $n$ is unknown and $\mu=1$.

By substituting (16) in autonomous differential equations, and assuming $\mu=1$ we get

$$
\begin{aligned}
x^{\prime} & =-1-z-3 y+x^{2}-x z+w \\
y^{\prime} & =\frac{x y z}{2 n(2 n+1)[y+(2 n+1) z]}-y(2 z-4-x) \\
z^{\prime} & =-\frac{x y z}{2 n(2 n+1)[y+(2 n+1) z]}-2 z(z-2) \\
w^{\prime} & =-2 z w+x w
\end{aligned}
$$

also,

$$
\omega_{\text {eff }}=-1-\frac{2 \dot{H}}{3 H^{2}}=-\frac{1}{3}(2 z-1)
$$

There are 8 real critical points of this system. We will now do the detailed stability and acceleration analysis for all the points. Plot legends 1, 2, 3, etc have been used to denote different eigenvalue of 
particular critical point.

$\mathbf{P}_{1}:(-4,5,0,0) . \quad \Omega_{m}=0, \Omega_{r}=0, \omega_{e f f}=\frac{1}{3}$. Eigenvalues of this critical point are $-3,-4,-5$ and $\frac{2\left(4 n^{2}+2 n-1\right)}{n(2 n+1)}$. The eigenvalues of this critical point is converging to 4 . This point is stable for only the small range of $n\left(n<\frac{\sqrt{5}-1}{4}\right)$. Since the value of $\omega_{\text {eff }}$ is positive, acceleration for this model is could not possible.

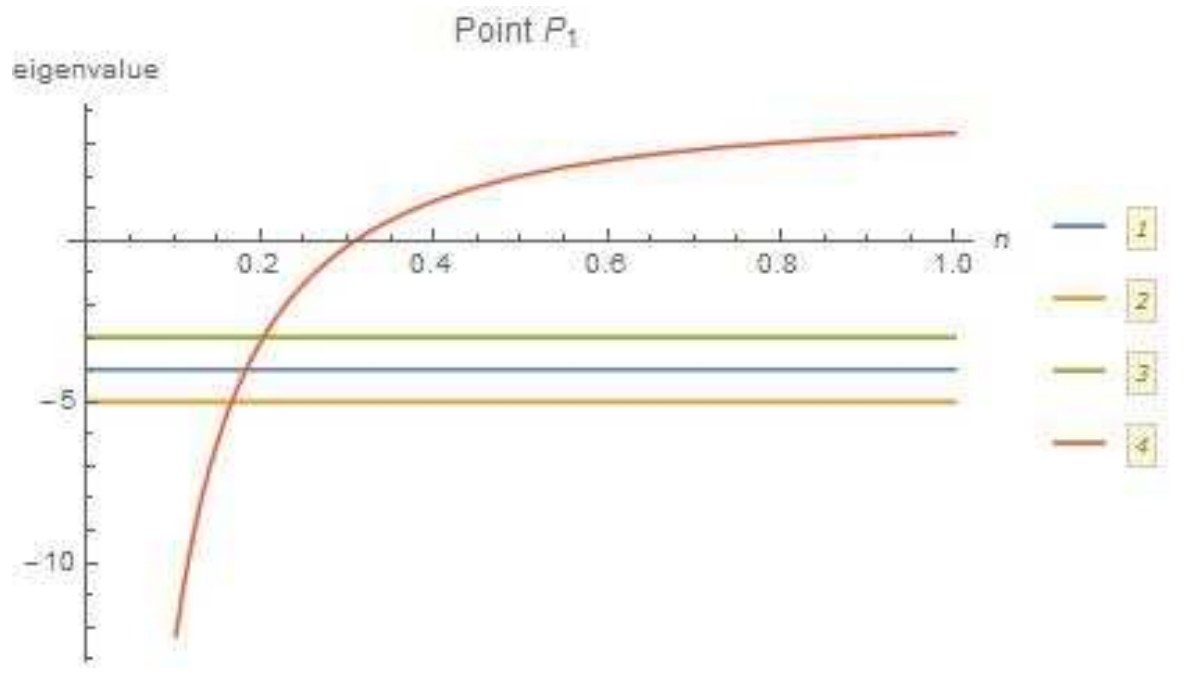

$\mathbf{P}_{\mathbf{2}}:(0,-1,2,0) . \Omega_{m}=0, \Omega_{r}=0, \omega_{e f f}=-1$. We here observe that real part of all the eigenvalues are negative for $0.152379 \leq n<0.19908$. Hence this point is spiral stable for a small range. Apart from that $\omega_{e f f}$ is negative, hence this point gives acceleration. This point is completely dominated by geometric curvature as $\Omega_{G C}=1$. Since there is no matter or radiation component in this point, this point could be considered to be responsible for late time acceleration of the universe.

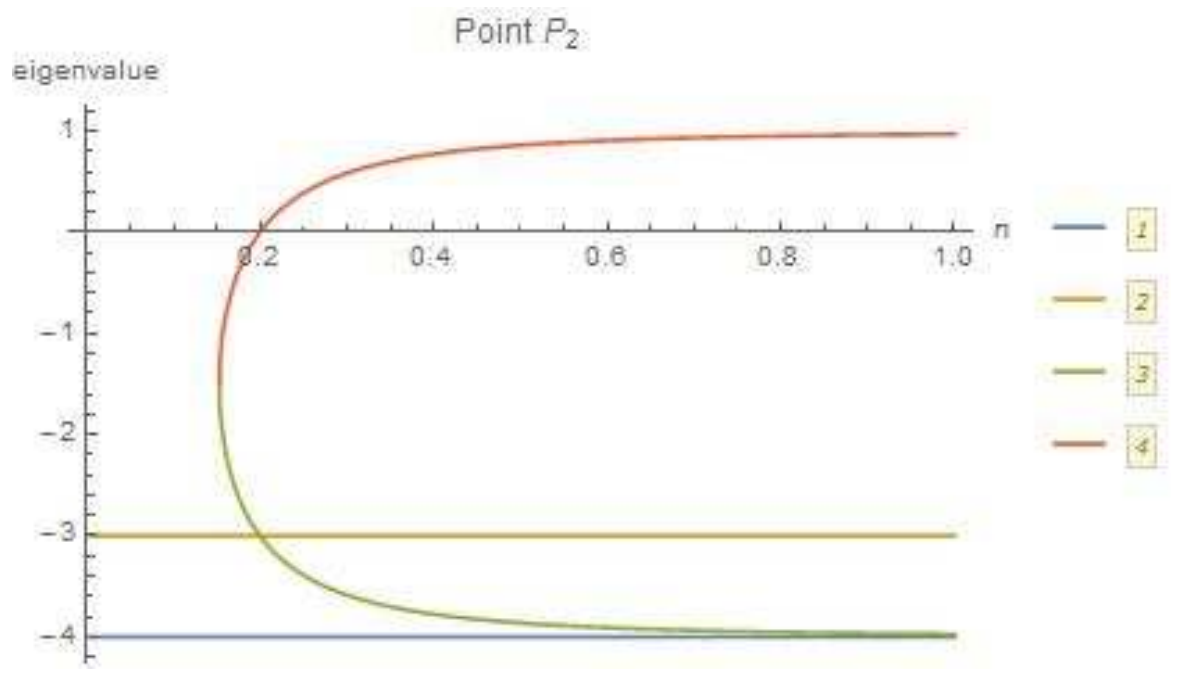

$\mathbf{P}_{\mathbf{3}}:(-1,0,2,0) . \Omega_{m}=0, \Omega_{r}=0, \omega_{\text {eff }}=-1$. Eigenvalues of this critical point are: $-5,-4,-4$, $\frac{-1-2 n-8 n^{2}-8 n^{3}}{2 n(1+2 n)^{2}}$. This model is stable for $n>\frac{1}{24}(-8+\sqrt[3]{(928-96 \sqrt{93}}+2 * 2 \sqrt{2 / 3} \sqrt[3]{29+3 \sqrt{93}})$. This model could provides acceleration since $\omega_{e f f}$ is negative. Also we note here that this critical point is a 'stable proper node' as two distinct eigenvectors arises for repeated eigenvalues. Like $P_{2}$, this point is also 
completely dominated by geometric curvature but for a large range of $n$ value which is evident from the plot below.

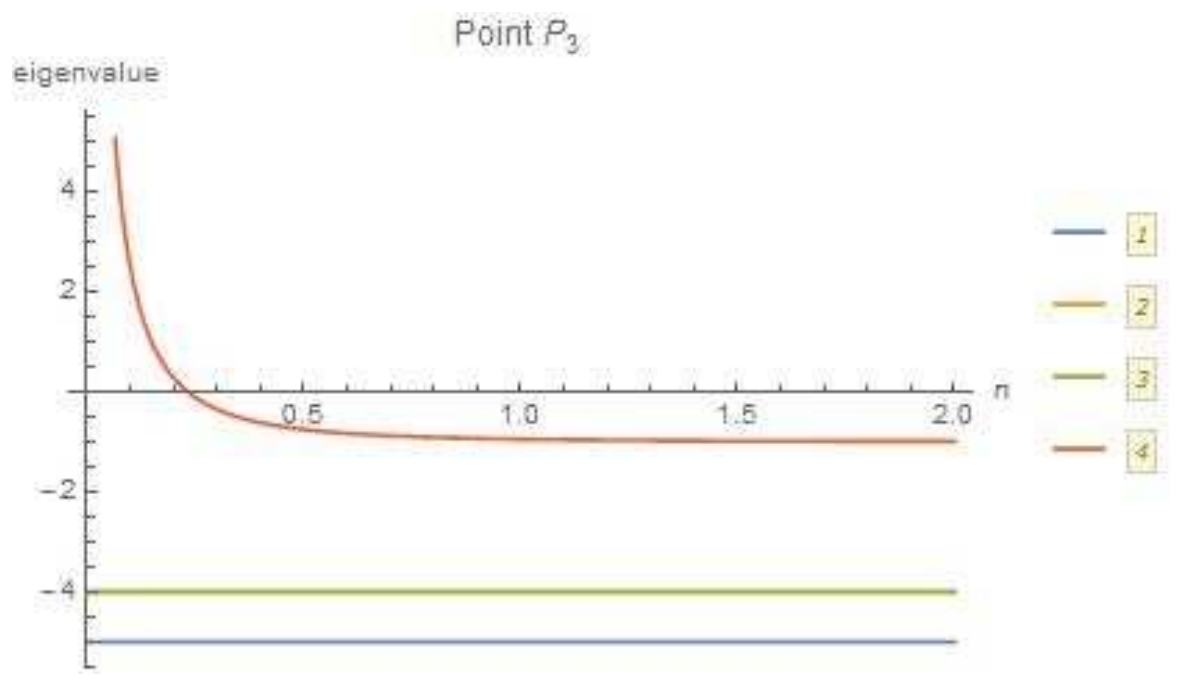

$\mathbf{P}_{4}:(3,0,2,0) . \quad \Omega_{m}=-4, \Omega_{r}=0, \omega_{e f f}=-1$. Eigenvalues of this critical point are: $-4,-1,4$, $\frac{3\left(1+2 n+8 n^{2}+8 n^{3}\right)}{2 n(1+2 n)^{2}}$. It is clear that since one eigenvalue is positive, this point is not stable and acceleration occurs for this point.

$\mathbf{P}_{5}:(4,0,2,-5) . \Omega_{m}=0, \Omega_{r}=-5, \omega_{e f f}=-1$. Eigenvalues of this critical point are: $-4,-1,5$, $\frac{2\left(1+2 n+8 n^{2}+8 n^{3}\right)}{2 n(1+2 n)^{2}}$. Again here one eigenvalue is positive hence this point is not stable and acceleration occurs for this point as $\omega_{e f f}=-1$.

$\mathbf{P}_{6}:\left(-\frac{12\left(n^{2}+2 n^{3}\right)}{-1+2 n+4 n^{2}}, \frac{\left(-1+2 n+8 n^{2}+8 n^{3}\right)\left(1-2 n+8 n^{2}+24 n^{3}\right)}{2\left(-1+2 n+4 n^{2}\right)^{2}}, \frac{-1+2 n-8 n^{2}-24 n^{3}}{2\left(-1+2 n+4 n^{2}\right)}, 0\right)$. $\Omega_{m}=\frac{1-4 n-18 n^{2}+16 n^{3}+104 n^{4}+16 n^{5}-96 n^{6}}{\left(-1+2 n+4 n^{2}\right)^{2}}, \Omega_{r}=0, \omega_{e f f}=\frac{4 n^{2}+8 n^{3}}{-1+2 n+4 n^{2}}$. From the value of $\omega_{e f f}$ we see that the acceleration for this model occurs from $\frac{1}{72}\left(-16+\sqrt[3]{7136-288 \sqrt{597}}+2^{\frac{5}{3}} \sqrt[3]{223+9 \sqrt{597}}\right)<n<$ $\frac{1}{4}(\sqrt{5}-1)$. Also the real part of all the eigenvalues is negative in few regions for $0<n<1.5$ as shown in the figure. So for this model, the stability and acceleration can occur simultaneously but it is in very small region. Since the value of $\Omega_{m}$ for this point is non zero, this point can be used for the matter-dominated epoch. This point does not govern acceleration and stability for a long duration which makes it a better candidate for matter dominated point. 


\section{Point $P_{6}$}

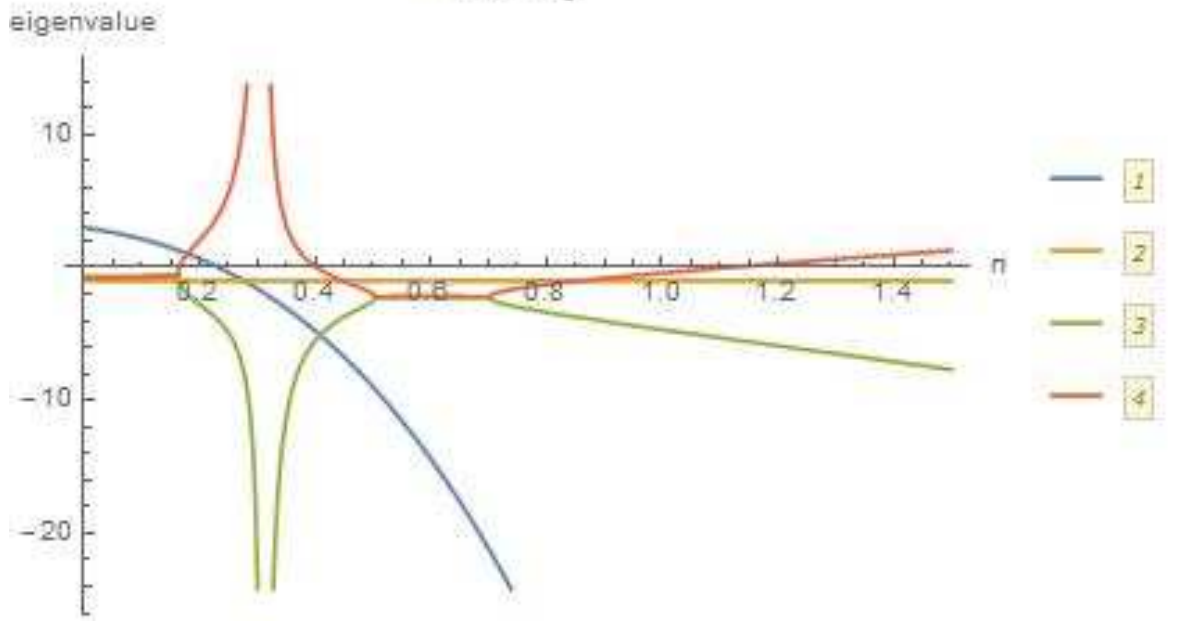

$\mathbf{P}_{\boldsymbol{7}}:\left(-\frac{16\left(2 n^{3}+n^{2}\right)}{4 n^{2}+2 n-1}, \frac{8\left(16 n^{6}+24 n^{5}+12 n^{4}-n^{2}\right)}{\left(4 n^{2}+2 n-1\right)^{2}},-\frac{8\left(2 n^{3}+n^{2}\right)}{4 n^{2}+2 n-1}, \frac{-128 n^{6}+112 n^{4}+16 n^{3}-20 n^{2}-4 n+1}{\left(4 n^{2}+2 n-1\right)^{2}}\right)$. Two of four eigenvalues of this point are 1 and $-4\left(-1+2 n+8 n^{2}+8 n^{3}\right)$. The second eigenvalue would be negative for $n>\frac{1}{24}\left(-8+\sqrt[3]{(928-96 \sqrt{93}}+2 * 2^{2 / 3} \sqrt[3]{29+3 \sqrt{93}}\right)$ i.e. $n>0.2328$. Also the real part of third and fourth eigenvalue are negative for small n. Hence we do not get any region were all the other three eigenvalues are positive. Hence this would be a saddle point due to opposite signs of eigenvalues. Moreover, this point could provide acceleration for, $\frac{1}{48}(-12+\sqrt[3]{3456-192 \sqrt{321}}+4 \sqrt[3]{3(18+\sqrt{321}})<n<\frac{1}{4}(\sqrt{5}-1)$ which is again very short lived.

$\mathbf{P}_{\mathbf{8}}:\left(-\frac{2\left(-1+2 n+12 n^{2}+16 n^{3}\right)}{1-2 n+8 n^{3}}, \frac{\left(-1+2 n+8 n^{2}+8 n^{3}\right)\left(-1+2 n+24 n^{2}+40 n^{3}\right)}{4 n^{2}\left(1-4 n^{2}+8 n^{3}+16 n^{4}\right)}, \frac{-1+4 n+24 n^{2}-16 n^{3}-176 n^{4}-160 n^{5}}{4 n^{2}\left(1-4 n^{2}+8 n^{3}+16 n^{4}\right)}, 0\right)$. The $\omega_{\text {eff }}$ here suggests that the model does not give any acceleration for $n>0$. But this model becomes stable in $n>\sqrt{96 n^{6}-16 n^{5}-104 n^{4}-16 n^{3}+18 n^{2}+4 n-1}$ i.e near $n=1.11569$, which is evident from the plot.

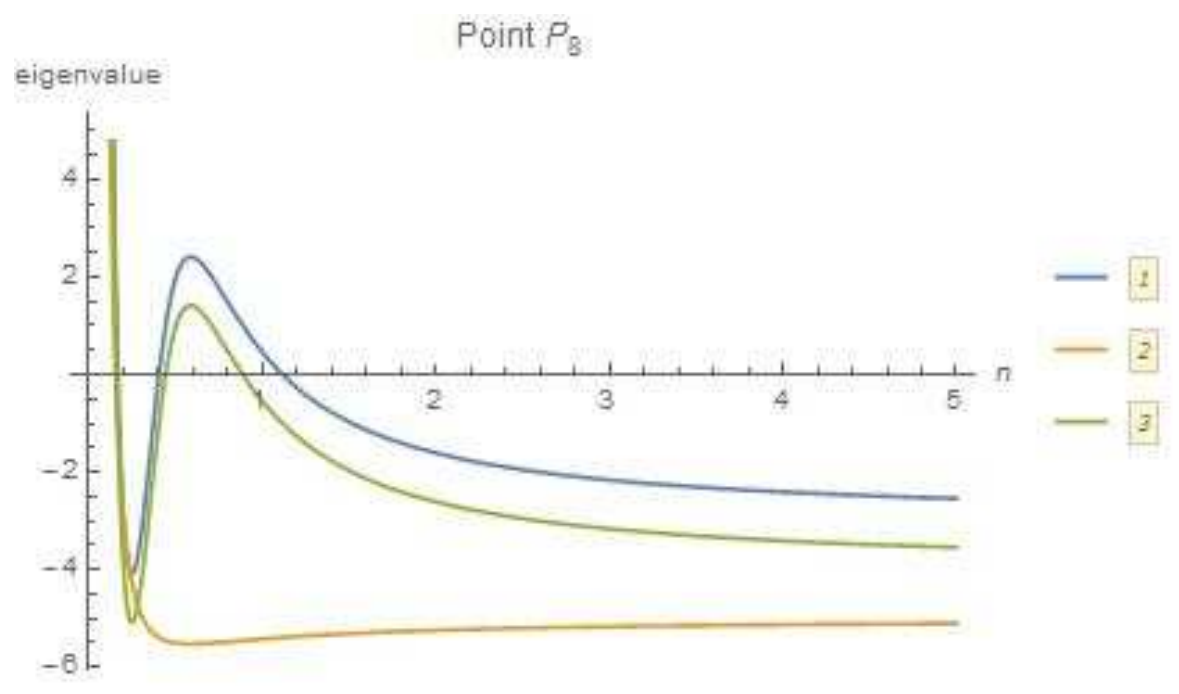

Case B: $\mu$ is unknown and $n=1$.

As a second case, we follow a different approach, i.e. by evaluating these equations for different values of $n(n>0)$ and $\mu$ being an unknown variable and study the dynamics of the model. The set of autonomous differential equations for $n=1$ would be as follows: 


$$
\begin{aligned}
x^{\prime} & =-1-z-3 y+x^{2}-x z+w \\
y^{\prime} & =-\frac{x y^{3} z \mu^{2}}{6(y+z)^{3}}-y(2 z-4-x) \\
z^{\prime} & =\frac{x y^{3} z \mu^{2}}{6(y+z)^{3}}-2 z(z-2) \\
w^{\prime} & =-2 z w+x w
\end{aligned}
$$

There are 5 real critical points of this system. Their detailed stability and acceleration analysis would be done.

$\mathbf{P}_{\mathbf{9}}:(-4,5,0,0) . \Omega_{m}=0, \Omega_{r}=0, \omega_{e f f}=\frac{1}{3}$. Eigenvalues are $-5,-4,-3$ and $4-\frac{2 \mu^{2}}{3}$. This point does not show any acceleration but stability occurs for $0<\mu<\sqrt{6}$. This point is similar to $P_{1}$.

$\mathbf{P}_{10}:(0,-1,2,0) . \quad \Omega_{m}=0, \Omega_{r}=0, \omega_{\text {eff }}=-1$. Eigenvalues are $-4,-3, \frac{1}{6}\left(-9-\sqrt{3} \sqrt{75-8 \mu^{2}}\right)$ and $\frac{1}{6}\left(-9+\sqrt{3} \sqrt{75-8 \mu^{2}}\right)$. This point always shows an acceleration and stability occurs only for $\sqrt{6}<\mu \leq \frac{5 \sqrt{\frac{3}{2}}}{2}$. This point is similar to $P_{2}$.

$\mathbf{P}_{11}:(-1,0,2,0) . \Omega_{m}=0, \Omega_{r}=0, \omega_{e f f}=-1$. Eigenvalues are $-5,-4,-4$ and -1 . Since $\omega_{\text {eff }}=-1$ this model behaves as a $\Lambda \mathrm{CDM}$ model. Also the eigenvectors of the repeated eigenvalues are independent hence this point is stable proper node. It is very interesting to note here that $P_{11}$ and $P_{3}$ are same and their stability behaviour is also same in both the cases.

$\mathbf{P}_{12}:(3,0,2,0) . \Omega_{m}=-4, \Omega_{r}=0, \omega_{e f f}=-1$. Eigenvalues are $-4,4,3$ and -1 . This point is similar to $P_{4}$. It is shown here to note that similarly like $P_{4}, P_{12}$ is also not stable. So linear approximation may give correct understanding for some critical points. But as we will note later that value of $n$ plays a crucial role.

$\mathbf{P}_{13}:(4,0,2,-5) . \Omega_{m}=0, \Omega_{r}=-5, \omega_{e f f}=-1$. Eigenvalues are $5,-4,4$ and 1 . This point does have acceleration but stability does not occurs due to opposite signs of eigenvalues. This point is similar to $P_{5}$.

As mentioned we will study the same critical point which were common in both the above mentioned cases. In this section, we will plot 3D figures to study the stability. Before that we will mention the set of autonomous differential equations for a general case as follows:

$$
\begin{aligned}
x^{\prime} & =-1-z-3 y+x^{2}-x z+w \\
y^{\prime} & =\frac{x y z \mu^{2 n}}{2 n(2 n+1)[y+(2 n+1) z]}-y(2 z-4-x) \\
z^{\prime} & =-\frac{x y z \mu^{2 n}}{2 n(2 n+1)[y+(2 n+1) z]}-2 z(z-2) \\
w^{\prime} & =-2 z w+x w
\end{aligned}
$$

It is almost impossible to find the general eigenvalues of this system autonomous differential equations. Since, all the points $P_{9}$ to $P_{13}$ were repeated critical points, they could be considered as some of the many critical point of the system (20). Then for each of the case, we do the stability analysis. Since here we have 2 parameters as $\mu$ and $n$ to study the stability, we first construct a 3D plot to show the behavior of eigenvalues for various $n$ and $\mu$. Later the region where the particular eigenvalue is negative could also be shown. This makes the analysis very clear. 
$\mathbf{P}_{9}:(-4,5,0,0) . \quad \Omega_{m}=0, \Omega_{r}=0, \omega_{e f f}=\frac{1}{3}$. Eigenvalues are $-5,-4,-4$ and $-\frac{2\left(-4 n^{2}+\mu^{2 n}-2 n\right)}{n(2 n+1)}$. Since $\omega_{\text {eff }}=-1$ this model behaves as a $\Lambda$ CDM model. Also the eigenvectors of the repeated eigenvalues are independent hence this point is stable proper node. From the figure, we can see that this point represent stability.

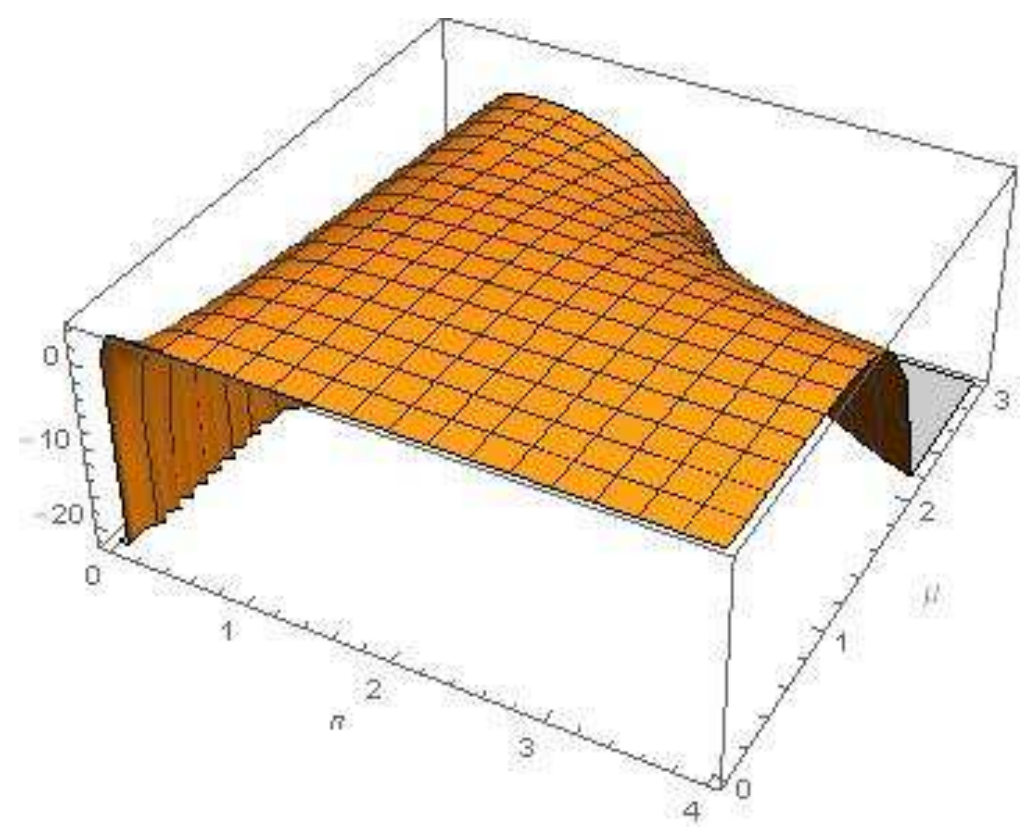

We now show a 2D plot with $n$ as $\mathrm{x}$-axis and $\mu$ as $\mathrm{y}$-axis to show the region where the eigenvalue $-\frac{2\left(-4 n^{2}+\mu^{2 n}-2 n\right)}{n(2 n+1)}$ is negative. The shaded portion shows that the eigenvalue is negative and critical point is stable for the particular combination of $n$ and $\mu$ as soon below. 


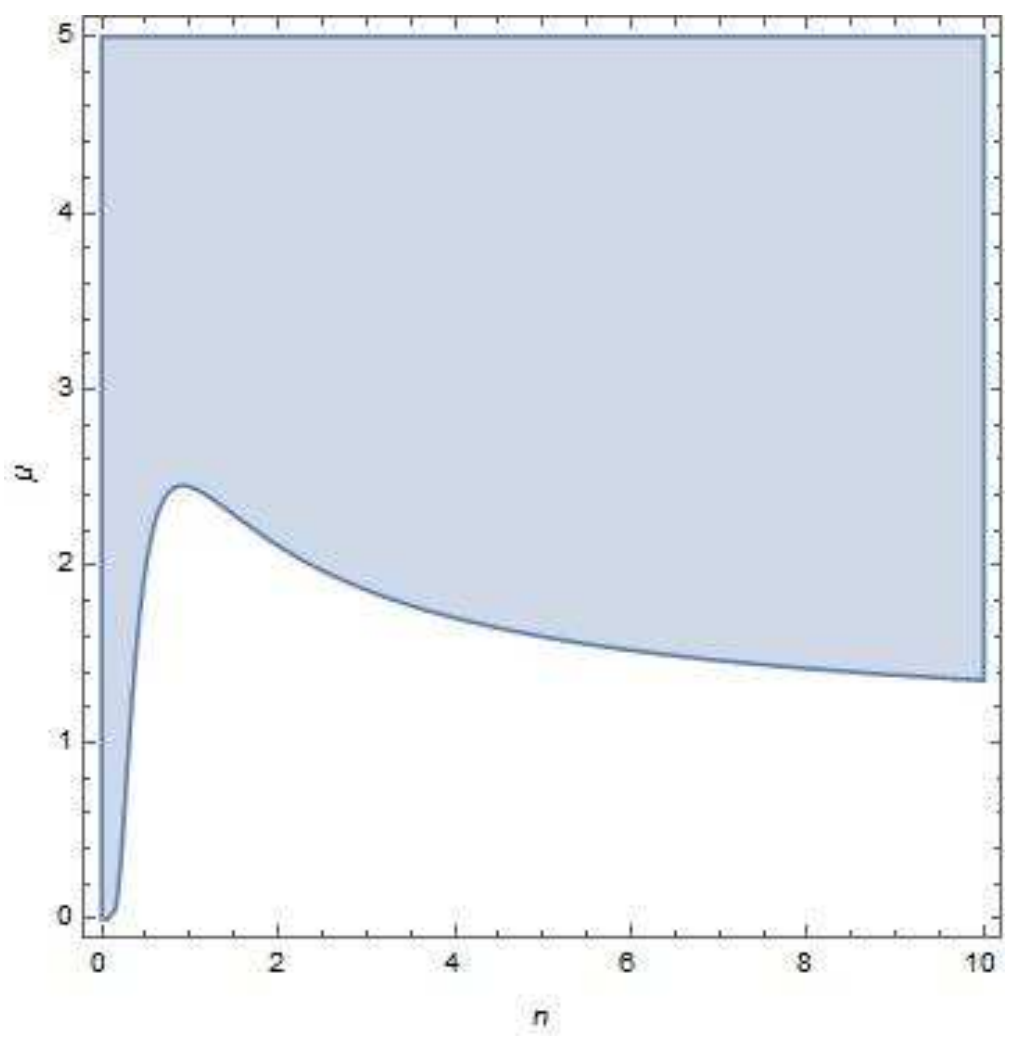

$\mathbf{P}_{10}:(0,-1,2,0) . \Omega_{m}=0, \Omega_{r}=0, \omega_{e f f}=-1$. Two of the eigenvalues are -3 and -4 . Plot for other two is shown below which shows the stability for considered values of $\mu$ and $n$. In the plot yellow colour represents the real part of the eigenvalue $\frac{-3 n-24 n^{3}-18 n^{2}-\sqrt{1600 n^{6}+2400 n^{5}+1300 n^{4}-64 n^{3} \mu^{2 n}+300 n^{3}-48 n^{2} \mu^{2 n}+25 n^{2}-8 n \mu^{2 n}}}{2 n(2 n+1)(4 n+1)}$ and blue represents the real part of eigenvalue $\frac{-3 n-24 n^{3}-18 n^{2}+\sqrt{1600 n^{6}+2400 n^{5}+1300 n^{4}-64 n^{3} \mu^{2 n}+300 n^{3}-48 n^{2} \mu^{2 n}+25 n^{2}-8 n \mu^{2 n}}}{2 n(2 n+1)(4 n+1)}$

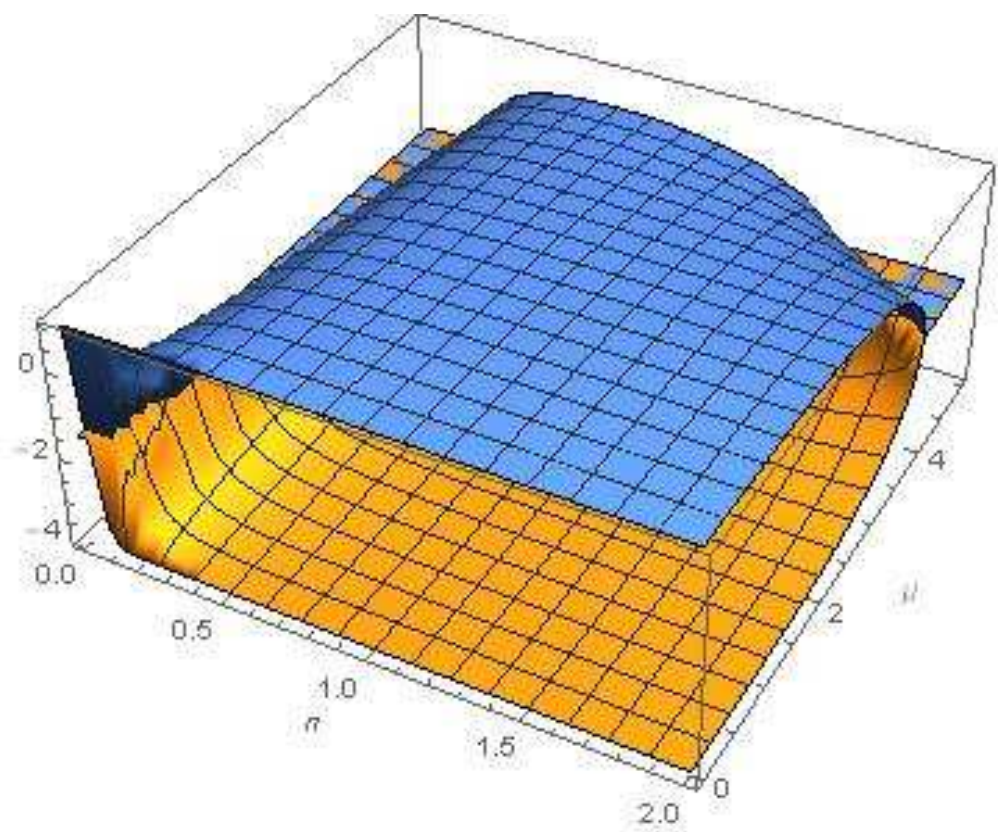

We again look at the $2 \mathrm{D}$ plot to show the region where both the eigenvalues are negative. The shaded portion in the below figure represents stability. 


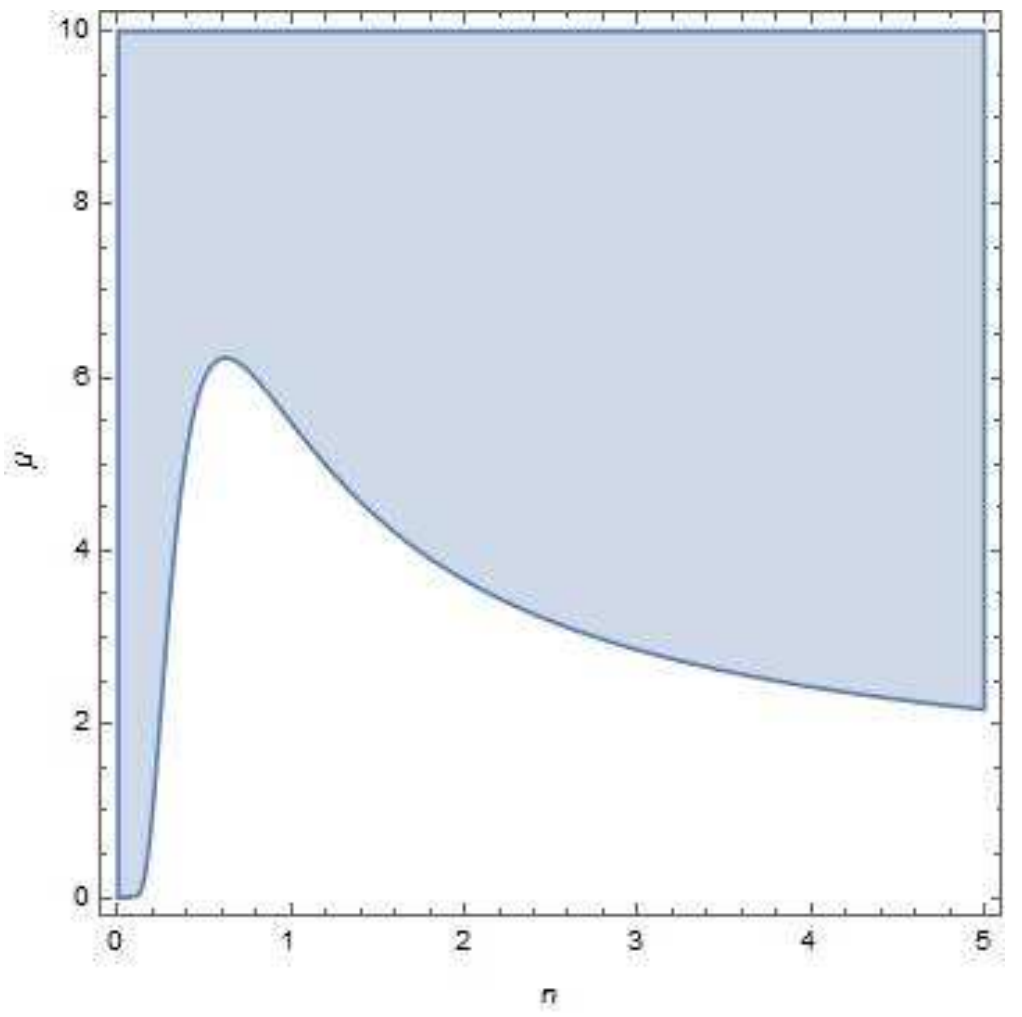

$\mathbf{P}_{11}:(-1,0,2,0) . \Omega_{m}=0, \Omega_{r}=0, \omega_{e f f}=-1$. Eigenvalues are $-5,-4,-4$ and $\frac{-8 n^{3}-8 n^{2}+\mu^{2 n}-2 n}{2 n(2 n+1)^{2}}$. The eigenvectors of the repeated eigenvalues are independent hence this point is stable proper node. We can see that the stability do occur for some combinations of $n$ and $\mu$. This is also evident from the $2 \mathrm{D}$ plot shown between $n$ and $\mu$.

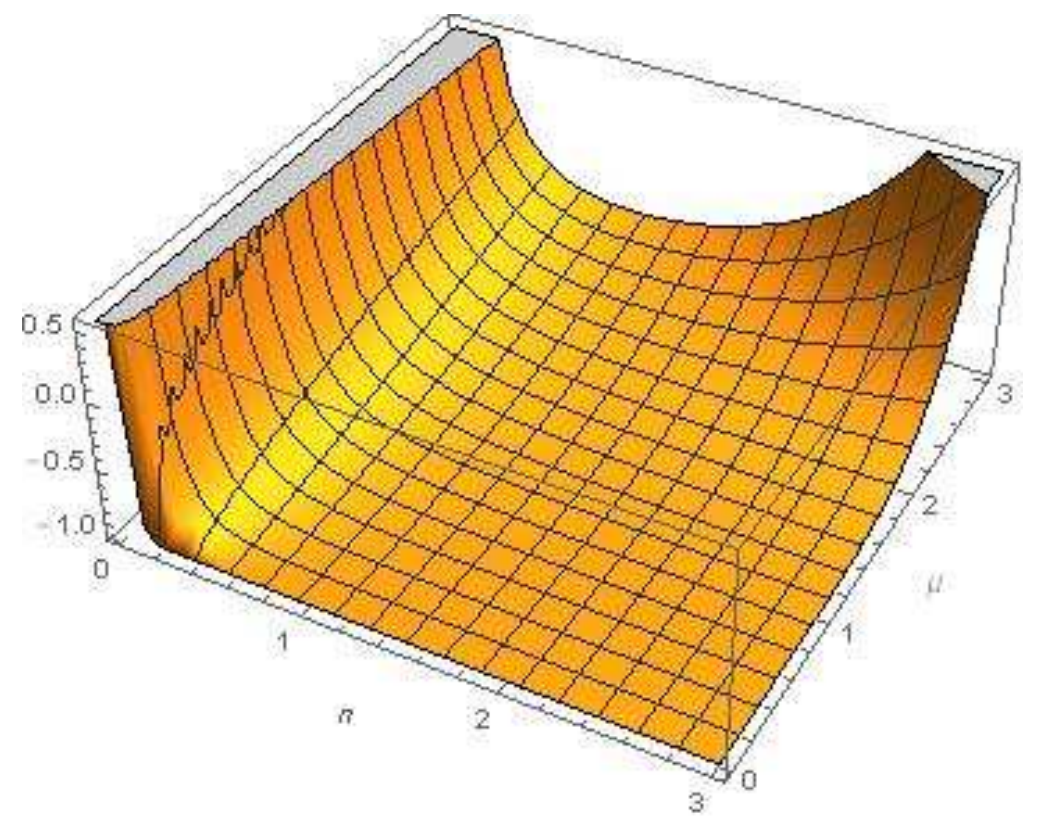




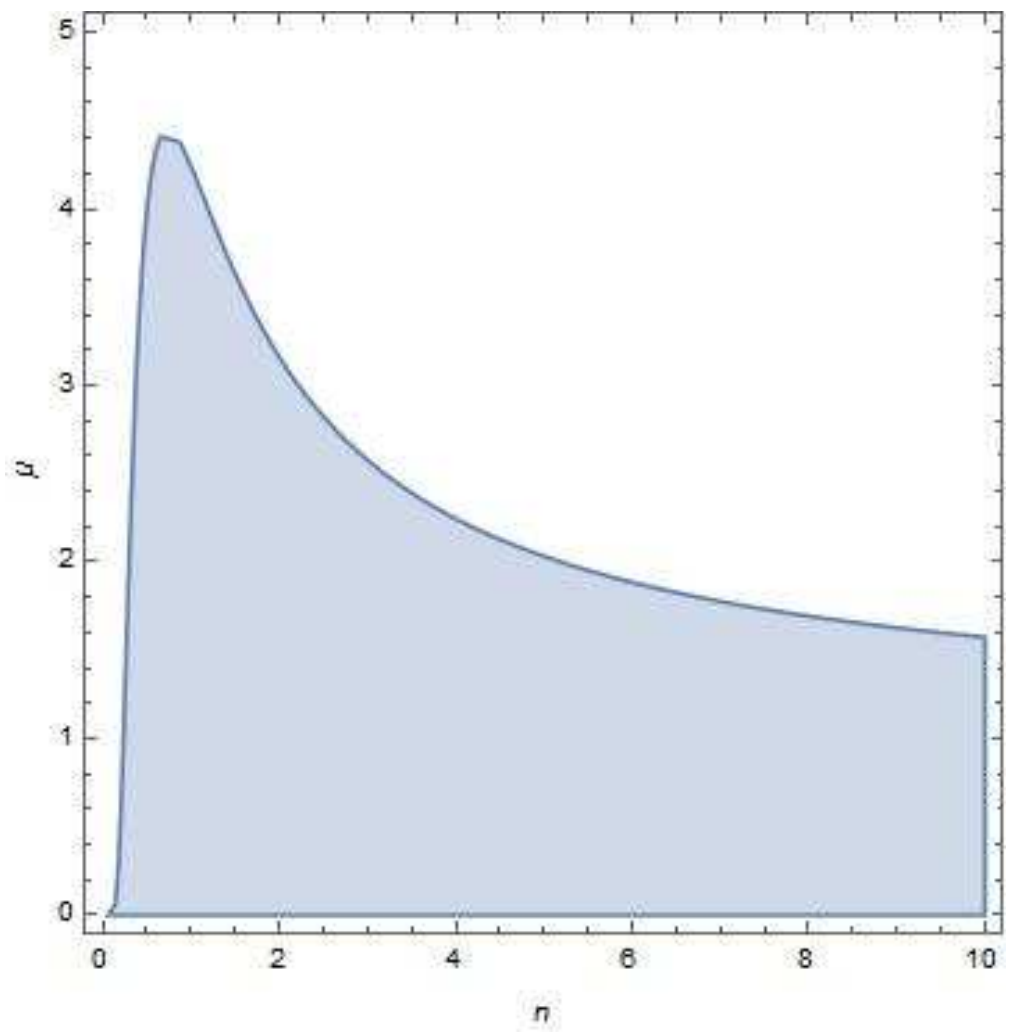

$\mathbf{P}_{12}:(3,0,2,0) . \Omega_{m}=-4, \Omega_{r}=0, \omega_{e f f}=-1$. Eigenvalues are $-4,4,-1$ and $-\frac{3\left(-8 n^{3}-8 n^{2}+\mu^{2 n}-2 n\right)}{2 n(2 n+1)^{2}}$. This point is similar to $P_{4}$. It can be noted here that similarly like $P_{4}, P_{12}$ is also not stable. Signs of eigenvalues are opposite hence stability could not be achieved. The plot of the eigenvalue $-\frac{3\left(-8 n^{3}-8 n^{2}+\mu^{2 n}-2 n\right)}{2 n(2 n+1)^{2}}$ in $n-\mu$ plane is as follows:

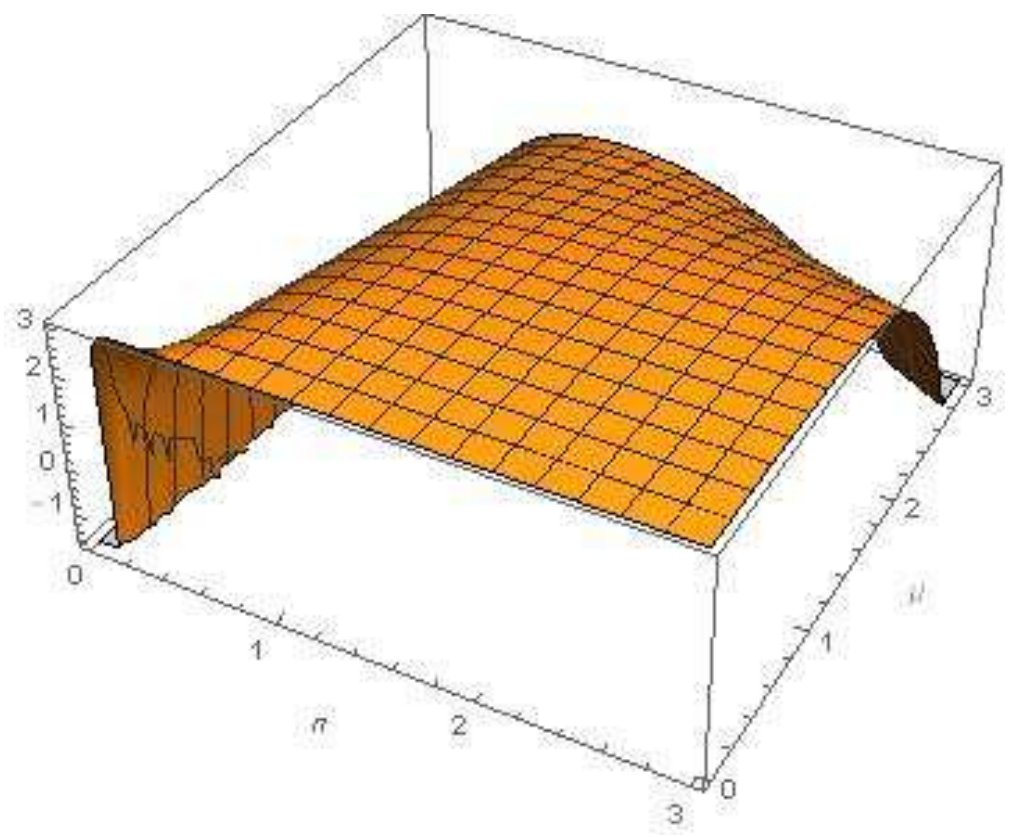




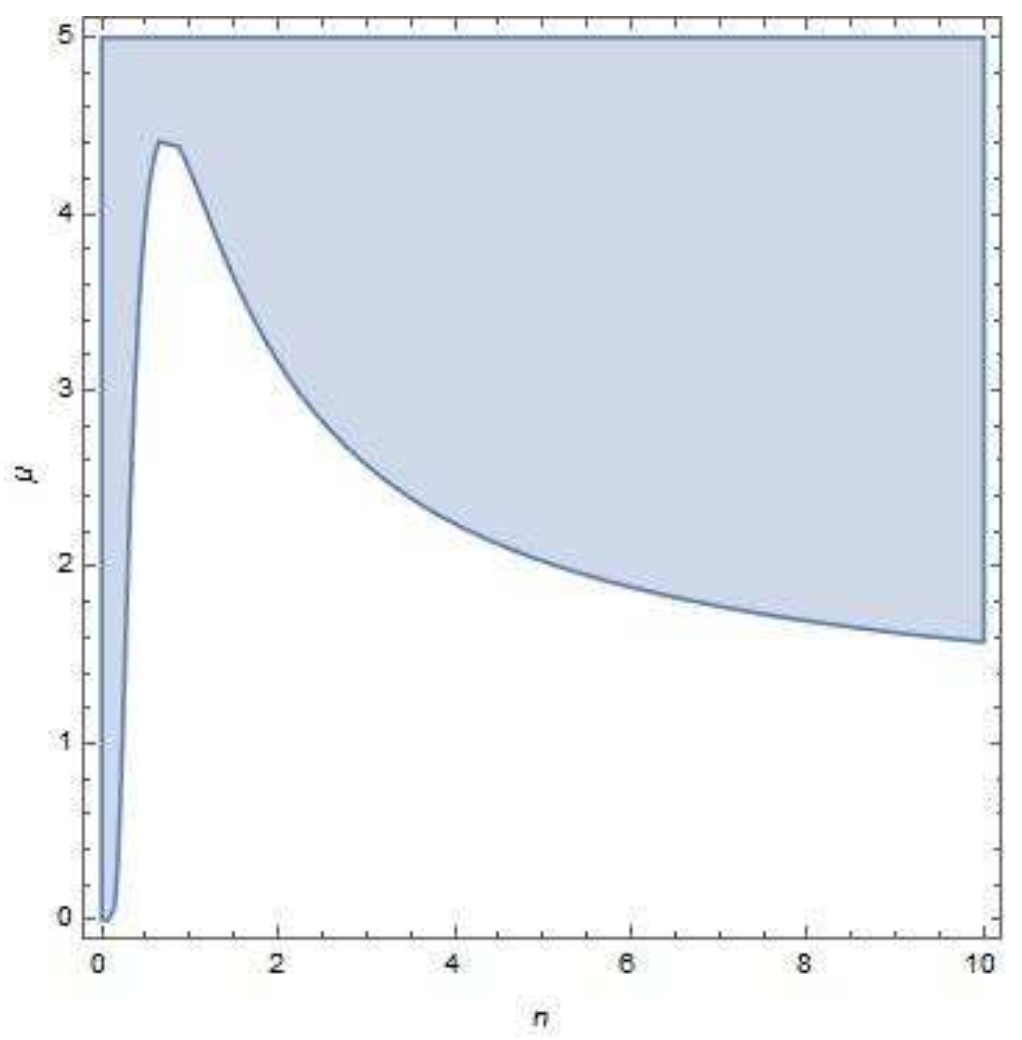

$\mathbf{P}_{13}:(4,0,2,-5) . \quad \Omega_{m}=0, \Omega_{r}=-5, \omega_{e f f}=-1$. Eigenvalues are 5, -4, 4 and 1. This point shows acceleration due to negative value of $\omega_{\text {eff }}$ but stability does not occurs due to opposite signs of eigenvalues. This point is similar to $P_{5}$.

We note a peculiar thing here that whenever $x+y+z=1$, we have a stability era which are attained in $P_{9}, P_{10}$ and $P_{11}$. This indicates that the completely geometric curvature dependent universe would also bring the stability along with acceleration. It is seen from the observations that the dark energy is responsible for the stability of the universe. Here the geomtric curvature is playing the exact same role and hence giving the required stability for the critical points with $x+y+z=1$.

\section{Results and Conclusion}

In this work, the asymptotic behaviour of two different viable $f(R)$ model has been done. We first noted that $f(R)=R-\mu R_{c}\left[1-\left(R^{2} / R_{c}^{2}\right)^{-n}\right]$ is an asymptote for $f(R)=R-\mu R_{c} \frac{\left(R / R_{c}\right)^{2 n}}{\left(R / R_{c}\right)^{2 n}+1}$ and $f(R)=R-\mu R_{c}\left[1-\left(1+R^{2} / R_{c}^{2}\right)^{-n}\right]$ for $R \gg R_{c}$. The tool of dynamical system analysis is being used for this work. It begins by introducing a set of dimensionless variables for the corresponding field equations. Then the system of autonomous differential equations are formed for the $f(R)$ model under consideration. The universe is assumed to be composed only of matter and radiation with no interaction among them. The real and compatible critical points of the system were studied. The value of $\omega_{\text {eff }}$ gives the acceleration phase and signature of the eigenvalues of the Jacobian matrix of the corresponding critical point gives the stability analysis. This set of equations are to be solved for 4 variables. This work was done in two different approaches. As a part of Case-A, we fixed the value of $\mu$ and the calculations were carried out for all the values of $n$. This resulted in formation of 8 critical point. The brief analysis of the result is as follows: 


\begin{tabular}{|c|c|c|}
\hline Point & Stability & Acceleration \\
\hline$P_{1}$ & Stable for $0<n<\frac{(\sqrt{5}-1)}{4}$ & Never \\
\hline$P_{2}$ & Spiral Stable for $n \in[0.152379,0.19908)$ & Always \\
\hline$P_{3}$ & $\begin{array}{l}\text { Stable for } n>\frac{1}{24}(-8+\sqrt[3]{(928-96 \sqrt{93}} \\
\left.+2 * 2^{2 / 3} \sqrt[3]{29+3 \sqrt{93}}\right)\end{array}$ & Always \\
\hline$\overline{P_{4}}$ & Not Stable & Always \\
\hline$P_{5}$ & Not stable & Always \\
\hline$P_{6}$ & Stable for few regions in $0<n<1.5$ & $\begin{array}{c}\frac{1}{72}(-16+\sqrt[3]{7136-288 \sqrt{597}} \\
\left.+2^{\frac{5}{3}} \sqrt[3]{223+9 \sqrt{597}}\right) \\
<n<\frac{1}{4}(\sqrt{5}-1)\end{array}$ \\
\hline$P_{7}$ & Not stable & $\begin{array}{c}\frac{1}{48}(-12+\sqrt[3]{3456-192 \sqrt{321}} \\
+4 \sqrt[3]{3(18+\sqrt{321})} \\
<n<\frac{1}{4}(\sqrt{5}-1)\end{array}$ \\
\hline$P_{8}$ & $n>\sqrt{96 n^{6}-16 n^{5}-104 n^{4}-16 n^{3}+18 n^{2}+4 n-1}$ & Never \\
\hline
\end{tabular}

Here, point $P_{2}$ is spiral stable due to the presence of some complex eigenvalues with negative real parts but later we study that this point is indeed stable when analyzed in more general form. We now use a different approach as Case B, where we fix $n$ and evaluate critical points by varying $\mu$. That gives 5 real and compatible critical points.

\begin{tabular}{|c|c|c|}
\hline Point & Stability & Acceleration \\
\hline$P_{9}$ & Stable for $0<\mu<\sqrt{6}$ & Never \\
\hline$P_{10}$ & Stable for $\sqrt{6}<\mu \leq \frac{5 \sqrt{\frac{3}{2}}}{2}$ & Always \\
\hline$P_{11}$ & Stable & Always \\
\hline$P_{12}$ & Not Stable & Always \\
\hline$P_{13}$ & Not stable & Always \\
\hline
\end{tabular}

The main aim of this work was to analyze the critical points of the system of (20). It could be generalized that the common critical points of case A and case B are among the critical points of the system. System (20) is a general one and does not take any particular case which makes it most suitable to study the behavior. We then looked at the overview of these common points.

\begin{tabular}{|c|c|c|}
\hline Point & Stability & Acceleration \\
\hline$P_{1} \sim P_{9}$ & Stable for $2 n(2 n+1)<\mu^{2 n}$ & Never \\
\hline$P_{2} \sim P_{10}$ & Stable for $25 n\left(8 n^{2}+6 n+1\right)<8 \mu^{2 n}$ & Always \\
\hline$P_{3} \sim P_{11}$ & Stable for $\mu^{2 n}<2 n(2 n+1)^{2}$ & Always \\
\hline$P_{4} \sim P_{12}$ & Not Stable & Always \\
\hline$P_{5} \sim P_{13}$ & Not stable & Always \\
\hline
\end{tabular}

For these points, we showed the behavior of eigenvalue with respect to range of values of $\mu$ and $n$. Region where the eigenvalues become negative was also shown. We noted a very peculiar property about critical points that the stability occurs only for the points $P_{1} \sim P_{9}, P_{2} \sim P_{10}$ and $P_{3} \sim P_{11}$. All these points have a property that $x+y+z=1$. We also note a peculiar property about points $P_{4} \sim P_{12}$ and $P_{5} \sim P_{13}$. These points have $\Omega_{m}=-4$ and $\Omega_{r}=-5$ respectively which are highly exotic. But still $\omega_{\text {eff }}$ remains 
-1. This occurs due to the choice of the dynamical variable $x$. Also $P_{4} \sim P_{12}$ and $P_{5} \sim P_{13}$ are the only points for which the value of $x$ is positive. Here we can say that due to this positive value of $x$ geometric part has an anti-exotic kind of behavior and these are the only points for which stability never occurs. Unlike our previous work [12, this article describes the stability analysis for a more general model. Moreover the stability analysis and acceleration phase were analyzed for a particular case of the model $f(R)=R-\mu R_{c}\left(R / R_{c}\right)^{p}$ with $0<p<1, \mu, R_{c}>0$. Whereas in this article we discuss stability analysis for every possible values of our parameters $\mu$ and $n$. From all the above calculations and discussions, we could conclude that in the case of modified gravity (here $f(R)$ ) acceleration could be achieved by modifying the geometric components of the universe. Stability and acceleration phase are achieved which were otherwise achieved by exotic dark energy. It is obvious to note that $x+y+z=1$ means that geometric curvature dominated universe and we see that it is not just responsible for the acceleration of the universe but adding that function helps to get the stability of the universe which was otherwise achieved by dark energy. This work states the region where the stability of a particular point occurs. More models of such viable $f(R)$ could be constructed to extend this work. Apart from this some different category of modification like $f(R, G)$ or Scalar Tensor theory could be considered and the use of dynamical system analysis could be applied further and stability analysis for such cases could be carried out. Scalar fields could also be considered as one of the fluid in the universe to study its dynamics. Scalar fields act as a dark energy component and it would be interesting to see the effect of both the forms of modification in

the universe. Several forms of linear and non linear interactions in the fluids could also be studied with modification in the gravity.

\section{Acknowledgement}

Parth Shah, the author of this paper would like to acknowledge Department of Science and Technology (DST) for INSPIRE Fellowship (Ref. No. IF160358). He also thank the Mathematics department of BITS Pilani Goa campus for providing other necessary research facilities. The authors are very much thankful to the anonymous reviewer for his constructive comments to improve the quality of work.

\section{References}

[1] S. Perlmutter et. al., Astrophys. J. 517 (1999) 565.

[2] A. G. Riess et al., Astron. J. 116 (1998) 1009.

[3] S. Nojiri, S. D. Odintsov, V. K. Oikonomou, Phys. Rep. 692 (2017) 1.

[4] S. Nojiri and S. D. Odintsov, Int. J. Geom. Methods Mod. Phys. 04 (2007) 115.

[5] S. Capozziello, R. DAgostino, O. Luongo, Int. J. Mod. Phys. D 28 (2019) 1930016.

[6] T. P. Sotiriou, V. Faraoni, Rev. Mod. Phys. 82 (2010) 451.

[7] A. De Felice, S. Tsujikawa, Living Rev. Relativity 13 (2010) 3.

[8] L. Amendola, D. Polarski, S. Tsujikawa, Phys. Rev. Lett. 98 (2007) 131302.

[9] T. P. Sotiriou, Classical Quantum Gravity 23 (2006) 5117.

[10] Y.-S. Song, W. Hu, I. Sawicki, Phys. Rev. D 75 (2007) 044004.

[11] S. Nojiri, S. D. Odintsov, Phys. Rev. D 74 (2006) 086005. 
[12] P. Shah, G. C. Samanta, Eur. Phys. J. C. 79 (2019) 414.

[13] S. D. Odintsov, V. K. Oikonomou, Phys. Rev. D 96 (2017) 104049.

[14] K. Bamba, S. Nojiri, S. D. Odintsov, D. Sez-Gmez, Phys. Rev. D 90 (2014) 124061.

[15] S. Capozziello, M. De Laurentis, V. Faraoni, Open Astron. J. 3 (2010) 49.

[16] R. Gannouji, D. Polarski, A. Ranquet, A. A. Starobinsky, JCAP 09 (2006) 016.

[17] K. A. Bronnikov, A. A. Starobinsky, JETP 85 (2007) 1-5.

[18] K. A. Bronnikov, M. V. Skvortsova, A. A. Starobinsky, Gravitation and Cosmology 16 (2010) 216.

[19] K. A. Bronnikov, A. A. Starobinsky, Modern Physics Letters A 2420091559.

[20] P. G. Bergmann, Int. J. Theor. Phys. 1 (1968) 25.

[21] V. Faraoni, Cosmology in Scalar Tensor Gravity Kluwer Academic, Dordrecht (2004).

[22] Jr. N. Kenneth, Astrophys. J. 161 (1970) 1059.

[23] R. V. Wagoner, Phys. Rev. D 1 (1970) 3209.

[24] C. Brans and R. H. Dicke, Phys. Rev. 124 (1961) 925.

[25] R. H. Dicke, Phys. Rev. 125 (1962) 2163.

[26] S. Nojiri, S. D. Odintsov, et.al., Eur. Phys. J. C 79 (2019) 565.

[27] K. Bamba, C. Q. Geng, C. C. Lee and L. Wei, JCAP 1101 (2011) 021.

[28] K. Bamba, R. Myrzakulov, S. Nojiri, S. D. Odintsov, Phys. Rev. D 85 (2012) 104036.

[29] K. Bamba, C. Q. Geng, JCAP 11 (2011) 008.

[30] K. Bamba, S. Nojiri, S. D. Odintsov Phys. Lett. B 731 (2014) 257.

[31] K. Bamba, M. Jamil, D. Momeni, R. Myrzakulov, Astrophysics and Space Science 344 (2013) 259.

[32] K. Bamba, S. Nojiri, S. D. Odintsov Phys. Lett. B 725 (2013) 368.

[33] T. Harko, F. S. N. Lobo, S. Nojiri, and S. D. Odintsov, Phys. Rev. D 84 (2011) 024020.

[34] Z. Yousaf, K. Bamba, M. Z. H Bhatti, Phys. Rev. D 93 (2016) 124048.

[35] K. Bamba, S. D. Odintsov, L. Sebastiani, S. Zerbini, Eur. Phys. J. C 67 (2010) 295.

[36] D. Lovelock, J. Math. Phys. 12 (1971) 498.

[37] T. Padmanabhan, Rept. Prog. Phys. 73 (2010) 046901.

[38] L. Amendola, R. Gannouji, D. Polarski and S. Tsujikawa, Phys. Rev. D 75 (2007) 083504.

[39] Judit Prez-Romero and Savvas Nesseris, Phys. Rev. D 97 (2018) 023525.

[40] W. Hu and I. Sawicki, Phys. Rev. D 76 (2007) 064004. 
[41] A. A. Starobinsky, JETP Lett. 86 (2007) 157.

[42] S. Capozziello, M. De Laurentis and V. Faraoni, The Open Astronomy Journal 3 (2010) 49.

[43] S. Nojiri and S. D. Odintsov, Phys. Rept. 505 (2011) 59.

[44] K. Bamba, S. Capozziello, S. Nojiri and S. D. Odintsov, Astrophys. Space Sci. 342 (2012) 155.

[45] H. Motohashi, A. A. Starobinsky, and J. Yokoyama, Progress of Theoretical Physics, 123 (2010) 887.

[46] H. Motohashi, A. A. Starobinsky, and J. Yokoyama, JCAP 1106 (2011) 006.

[47] A. A. Starobinsky, JETP 86 (2007) 157.

[48] H. Motohashi, A. A. Starobinsky, J Yokoyama Int. J. Mod. Phys. D 20 (2011) 1347-1355.

[49] H. Motohashi, A. A. Starobinsky, J Yokoyama PRL 110 (2013) 121302.

[50] H. Motohashi, A. A. Starobinsky, Eur. Phys. J. C 77 (2017) 538.

[51] K. Bamba, S. Nojiri, S. D. Odintsov and D. Sáez-Gómez, Phys. Rev. D 90 (2014) 124061.

[52] K. Bamba, S. D. Odintsov and Petr V. Tretyakov, Eur. Phys. J. C 75 (2015) 344.

[53] S. Nojiri, S. D. Odintsov, V. K. Oikonomou, Phys. Rept. 692 (2017) 1.

[54] Z. Yousaf, K. Bamba and M. Zaeem-ul-Haq Bhatti, Phys. Rev. D 95 (2017) 024024.

[55] N. Godani and G. C. Samanta, Int. J. Mod. Phys. D 28 (2018) 1950039.

[56] S.D. Odintsov and V.K. Oikonomou, Phys. Rev. D 98 (2018) 024013.

[57] S. Capozziello, S. Nojiri and S. D. Odintsov, Phys. Lett. B 781 (2018) 106.

[58] S. D. Odintsov and V. K. Oikonomou, Annals Phys. 388 (2018) 267.

[59] N. Roy, arXiv: 1511.07978[gr-qc], 2015.

[60] S. D. Odintsov and V. K. Oikonomou, Phys. Rev. D 96 (2017) 104049.

[61] S. D. Odintsov, V. K. Oikonomou and Petr V. Tretyakov, Phys. Rev. D 96 (2017) 044022.

[62] M. Hohmann, L. Jarv and U. Ualikhanova, Phys. Rev. D 96 (2017) 043508.

[63] A. S. Bhatia and S. Sur, Int. J. Mod. Phys. D 26 (2017) 1750149.

[64] K. Bamba, D. Momeni and M. Al Ajmi, Eur. Phys. J. C 78 (2018) 771.

[65] S. Carneiro and H. Borges, Gen. Rel. Grav. 50 (2018) 1.

[66] S. Santos Da Costa et. al., Class. Quant. Grav. 35 (2018) 075013.

[67] P. Shah, G. C. Samanta and S. Capozziello, Int. J. Mod. Phys. A 33 (2018) 1850116.

[68] J. Wainright and G. F. R. Ellis, Dynamical Systems in Cosmology, (1997).

[69] A. A. Coley, Dynamical Systems and Cosmology, (2003). 
This figure "case1.jpg" is available in "jpg" format from: http://arxiv.org/ps/2111.09009v1 
This figure "case2.jpg" is available in "jpg" format from: http://arxiv.org/ps/2111.09009v1 
This figure "case4_2.jpg" is available in "jpg" format from: http://arxiv.org/ps/2111.09009v1 
This figure "case5_2.jpg" is available in "jpg" format from: http://arxiv.org/ps/2111.09009v1 
This figure "case6_2.jpg" is available in "jpg" format from: http://arxiv.org/ps/2111.09009v1 
This figure "case7_2.jpg" is available in "jpg" format from: http://arxiv.org/ps/2111.09009v1 
This figure "case8_2.jpg" is available in "jpg" format from: http://arxiv.org/ps/2111.09009v1 
This figure "case3.jpg" is available in "jpg" format from: http://arxiv.org/ps/2111.09009v1 
This figure "case4.jpg" is available in "jpg" format from: http://arxiv.org/ps/2111.09009v1 
This figure "case5.jpg" is available in "jpg" format from: http://arxiv.org/ps/2111.09009v1 
This figure "case6.jpg" is available in "jpg" format from: http://arxiv.org/ps/2111.09009v1 
This figure "case7.jpg" is available in "jpg" format from: http://arxiv.org/ps/2111.09009v1 
This figure "case8.jpg" is available in "jpg" format from: http://arxiv.org/ps/2111.09009v1 